\title{
Resonant tunneling through a two-level dot and double quantum dots
}

\author{
T. Pohjola ${ }^{1,2}$, J. König ${ }^{2}$, M. M. SalomaA ${ }^{1}$, J. Schmid $^{3}$, H. Schoeller ${ }^{2}$ and \\ GERD SCHÖN ${ }^{1,2}$ \\ 1 Materials Physics Laboratory, Helsinki University of Technology, 02150 Espoo, Finland \\ 2 Institut für Theoretische Festkörperphysik, Universität Karlsruhe, 76128 Karlsruhe, \\ Germany \\ 3 Max-Planck-Institut für Festkörperforschung, 70569 Stuttgart, Germany
}

(received 6 June 1997; accepted 10 September 1997)

PACS. 73.40Gk - Tunneling.

PACS. 72.15Qm- Scattering mechanisms and Kondo effect.

PACS. 73.20Dx - Electron states in low-dimensional structures.

\begin{abstract}
We study resonant tunneling through quantum-dot systems in the presence of strong Coulomb repulsion and coupling to the metallic leads. Motivated by recent experiments we concentrate on (i) a single dot with two energy levels and (ii) a double dot with one level in each dot. Each level is twofold spin-degenerate. Depending on the level spacing these systems are physical realizations of different Kondo-type models. Using a real-time diagrammatic formulation we evaluate the spectral density and the non-linear conductance. The latter shows a novel triple-peak resonant structure.
\end{abstract}

Quantum transport of electrons through discrete energy levels in quantum dots has been studied both experimentally $[1,2]$ and theoretically $[3,4,5,6,7,8]$. In small dots the Coulomb repulsion is strong and may yield the dominant energy scale, which gives rise to Coulomb blockade phenomena (see e.g. Refs. [4, 5, 6, 7, 8] for single dots and Refs. [9, 10, 11, 12] for multiple dots). At high temperatures, or when the coupling between the dots and metallic leads is weak, the electrons tunnel through the system sequentially. At low temperatures and for strong coupling to the leads resonant tunneling processes contribute significantly to the current $[3,13,14,15,16,17]$.

The purpose of this Letter is to investigate electronic transport phenomena through two ultrasmall quantum-dot systems: (i) a single dot with two energy levels and (ii) a double dot with a single level in each dot (see Fig. 1). We will show that the two models can be mapped onto each other. Thus, it is sufficient to concentrate on one, the single-dot model with two levels. In both cases we assume strong Coulomb repulsion between the electrons, such that we have to consider only two charge states, e.g. the empty or the singly-occupied dot system. We further allow for strong coupling between the quantum dot(s) and the metallic 
leads adjacent to them. This gives rise to resonant tunneling and - as has been shown for a single dot with one energy level [15, 16, 17] - nonequilibrium Kondo effects. In the following we consider the case with vanishing magnetic field. Accordingly the single-electron states are spin-degenerate, which is a prerequisite for Kondo effects. We suggest that the models could be realized experimentally in a setup resembling those in Refs. [18, 19, 20, 21, 22].

A systematic description of resonant tunneling phenomena has been developed in Refs. [3, $15,23]$ in terms of a real-time diagrammatic technique. It has been applied to the problems of electron transport through a single-level quantum dot as well as through a small metal island, both connected to reservoirs via tunnel junctions. In the quantum dot Kondo-type effects manifest themselves as a zero-bias maximum in the differential conductance. Furthermore, a zero-bias minimum has been predicted for the case where the level lies above the electrochemical potentials of the reservoirs [15]. Generalizing this method we calculate the spectral density of the two-level dot and the differential conductance for the current through the dot. As novel result we find a multiply peaked resonant structure both in the spectral functions and the differential conductance. When the two levels in the dot are positioned below the electrochemical potentials of the leads a triple-peak structure emerges in the nonlinear conductance: a zero-bias maximum with one satellite peak on each side. When the levels lie above the electrochemical potentials the peaks turn into notches. Depending on the level spacing our model interpolates between an $N=2$ and an $N=4$ Anderson model and the former corresponds to the $S=1 / 2$ Kondo model for a low lying level.

We denote the two energy levels of the dot by $\varepsilon_{+}$and $\varepsilon_{-}$(the choice of + and - arises from the eigenstates of the double dot system). Both levels are spin-degenerate, $\varepsilon_{i \uparrow}=$ $\varepsilon_{i \downarrow}=\varepsilon_{i}$ for $i \in\{+,-\}$. The Hamiltonian is a generalization of the Anderson model, $\mathcal{H}=\mathcal{H}_{0, \mathrm{D}}+\mathcal{H}_{0, \text { res }}+\mathcal{H}_{\mathrm{T}}$. The term $\mathcal{H}_{0, \mathrm{D}}=\sum_{i \sigma} \varepsilon_{i \sigma} c_{i \sigma}^{\dagger} c_{i \sigma}+U_{0} n_{\mathrm{D}}\left(n_{\mathrm{D}}-1\right)$ describes the isolated quantum dot. The interaction of the electrons in the dot is accounted for by $U_{0}$, with $n_{\mathrm{D}}=\sum_{i \sigma} c_{i \sigma}^{\dagger} c_{i \sigma}$ being the electron number. The electrons in the reservoirs are taken to be non-interacting, $\mathcal{H}_{0, \text { res }}=\sum_{\alpha \in\{\mathrm{R}, \mathrm{L}\}} \sum_{k i \sigma} \varepsilon_{\alpha k i \sigma} a_{\alpha k i \sigma}^{\dagger} a_{\alpha k i \sigma}$. The tunneling Hamiltonian $\mathcal{H}_{\mathrm{T}}=\sum_{\alpha k i \sigma}\left(T_{k i \sigma}^{\alpha} c_{i \sigma}^{\dagger} a_{\alpha k i \sigma}+\right.$ h.c.) describes tunneling between the dot and the reservoirs [24].

The double-dot system coupled in series between two reservoirs as shown in Fig. 1 with one spin-degenerate level in each dot is described by the Hamiltonian $\mathcal{H}=\mathcal{H}_{0, \mathrm{D}}+\mathcal{H}_{0, \mathrm{res}}+\mathcal{H}_{\mathrm{t}}+\mathcal{H}_{\mathrm{T}}$. The Hamiltonian of the two capacitively coupled dots is $\mathcal{H}_{0, \mathrm{D}}=\sum_{d \sigma} \varepsilon_{d \sigma} c_{d \sigma}^{\dagger} c_{d \sigma}+U_{12} n_{1} n_{\mathrm{r}}+$ $U_{0} \sum_{d} n_{d \uparrow} n_{d \downarrow}$. The index $d \in\{l, \mathrm{r}\}$ denotes the left or right dot, respectively. The term $\mathcal{H}_{\mathrm{t}}=\sum_{\sigma}\left(t c_{1 \sigma}^{\dagger} c_{\mathrm{r} \sigma}+\right.$ h.c. $)$ describes the tunneling between the dots, while the tunneling Hamiltonian $\mathcal{H}_{\mathrm{T}}$ is modified such that it only couples each dot to the lead adjacent to it.

The double-dot model can be mapped onto the two-level single-dot model in the regime of large charging energy, i.e., when $U_{0}$ and $U_{12}$ are larger than all other energy scales of the system. Then it is sufficient to consider only two adjacent charge states of the double dot. For appropriate values of a gate voltage coupled to the dots these states are the empty and the singly charged dot system. Then, we first solve the exact eigenstates of the double dot isolated from the leads, i.e., the eigenstates of $H_{0, \mathrm{D}}+H_{t}$. They are symmetric and antisymmetric combination of the single-dot states in the left and right-hand dots $|+\rangle=\alpha|l\rangle+\beta|r\rangle$ and $|-\rangle=\beta|l\rangle-\alpha|r\rangle$, with energies $\varepsilon_{+}$and $\varepsilon_{-}$, respectively. The tunneling $\mathcal{H}_{\mathrm{T}}$ couples the leads to these new eigenstates. Thus the effective tunneling matrix elements are $T_{\mathrm{L},+}=\alpha T, T_{\mathrm{R}_{\text {, }}}=$ $\beta T, T_{\mathrm{L}_{-}-}=\beta T, T_{\mathrm{R},-}=-\alpha T$. Except for this modification of the matrix elements the double-dot system coincides with the two-level single dot.

The mapping is not possible if we consider doubly-occupied states. The interaction between electrons in the same dot is stronger than between electrons in different dots. Hence two electrons in a double dot prefer to stay in different dots, and the low energy two-electron states 
are formed from these single-electron states only. In contrast in a single dot the two-electron states are composed of all single-electron states. By symmetry, in the case of three and four electron states the mapping is possible again.

The d.c. current through the quantum dot can be expressed as

$$
I=I_{\mathrm{L}}=-I_{\mathrm{R}}=-\frac{i e}{\hbar} \sum_{k i \sigma}\left(T_{k i \sigma}^{L}\left\langle a_{\alpha k i \sigma}^{\dagger} c_{i \sigma}\right\rangle-\text { H.c. }\right) \text {. }
$$

The statistical average of an arbitrary operator $O(t)$ can, quite generally, be expressed as

$$
\langle O(t)\rangle=\operatorname{tr}\left\{\rho_{0} T_{\mathrm{K}} \exp \left(-i \int_{\mathrm{K}} d t^{\prime} \mathcal{H}_{\mathrm{T}}\left(t^{\prime}\right)_{\mathrm{I}}\right) O(t)_{\mathrm{I}}\right\} .
$$

The time integration is performed along the Keldysh contour, i.e., forward in time from some initial time $t_{\mathrm{i}}$ to $t$ and then backward from $t$ to $t_{\mathrm{i}}$. The time-ordering operator $T_{\mathrm{K}}$ arranges operators with respect to this Keldysh contour. The subscript I denotes the interaction picture with respect to $\mathcal{H}_{0, \mathrm{D}}+\mathcal{H}_{0, \text { res }}$.

The leads are assumed to be large and in thermal equilibrium. Their electrochemical potentials are kept fixed at $\mu_{\mathrm{r}}=-e V_{\mathrm{r}}$ (for $\mathrm{r}=\mathrm{L}, \mathrm{R}$ ), and their electron distributions are described with Fermi functions. We are interested in the time evolution of the quantum states of the dot system, and therefore consider the reduced density matrix of the dot. To do this we first expand the exponential part in Eq. (2) into a series of the form

$T_{\mathrm{K}} \exp \left(-i \int_{\mathrm{K}} d t^{\prime} \mathcal{H}_{\mathrm{T}}\left(t^{\prime}\right)_{\mathrm{I}}\right)=\sum_{m=0}^{\infty}(-i)^{m} \cdot \int_{\mathrm{K}} d t_{1} \int_{\mathrm{K}} d t_{2} \cdots \int_{\mathrm{K}} d t_{m} T_{\mathrm{K}}\left\{\mathcal{H}_{\mathrm{T}}\left(t_{1}\right)_{\mathrm{I}} \mathcal{H}_{\mathrm{T}}\left(t_{2}\right)_{\mathrm{I}} \cdots \mathcal{H}_{\mathrm{T}}\left(t_{m}\right)_{\mathrm{I}}\right\}$

Here the times of the integrals are ordered with respect to the Keldysh contour, such that $t_{1}>t_{2}>\cdots>t_{m}$. The expectation value of this series is taken by tracing over the noninteracting reservoirs in the density matrices. We can perform the trace over the reservoir degrees of freedom exactly since the unperturbed Hamiltonian $\mathcal{H}_{0 \text {, res }}$ is bilinear in the leadelectron operators. These facts allow us to apply Wick's theorem, and the forward and backward propagators become coupled due to the trace over the leads. In Refs. [3, 15, 23] a systematic diagrammatic technique has been developed for presenting the terms of the expansion Eq. (3). There are well-defined rules for evaluating an arbitrary diagram and it is possible to identify and study processes of different orders.

The current can be expressed in terms of the spectral densities $A_{i \sigma}(\omega)$ of the electron states in the dot as [25]

$$
I=\sum_{i, \sigma} \frac{e}{\hbar} \int_{-\infty}^{\infty} d \omega \frac{\Gamma_{i \sigma}^{R} \Gamma_{i \sigma}^{L}}{\Gamma_{i \sigma}^{R}+\Gamma_{i \sigma}^{L}} \cdot\left[f\left(\omega-\mu_{\mathrm{R}}\right)-f\left(\omega-\mu_{\mathrm{L}}\right)\right] A_{i \sigma}(\omega)
$$

The factors $\Gamma_{i \sigma}^{\alpha}=2 \pi \sum_{k}\left|T_{k i \sigma}^{\alpha}\right|^{2} \delta\left(E-\varepsilon_{\alpha k i \sigma}\right)$ describe the coupling between the dot level $i$ and the lead $\alpha$ and they are assumed to be independent of energy [24]. The spectral function describes the spectrum of possible excitation of the system. In general there exist separate Green's and spectral functions for the four states of the dot: one for each spin of each level. Here we restrict ourselves to the zero-field case such that the levels and the corresponding spectral functions are spin-degenerate.

In general, we cannot sum up all possible contribution from the expansion Eq. (3). Similar as in Ref. [3, 13, 15] we proceed in a conserving approximation, taking into account non-diagonal matrix elements of the total density matrix up to the difference of one electron-hole pair excitation in the leads. In this case we find a set of integral equations for the spectral 
densities $A_{i}(\omega)$. While the coupling between the dot and the reservoirs is described within this approximation scheme, the double-dot states are solved exactly. This is reasonable when the coupling strength between the dots is larger than the coupling between the dots and the leads.

In the following, we choose a symmetrical bias $\mu_{\mathrm{L}}=-\mu_{\mathrm{R}}=e V / 2$ and consider equalstrength coupling of the dot to the leads $\Gamma_{\mathrm{L}}=\Gamma_{\mathrm{R}}$. The resulting conductance curves are symmetrical as a function of the voltage. The values for the energy levels that we mention, e.g., in the context of the illustrations, refer to the level positions before the renormalization due to tunneling. Finally, we choose the temperature $k_{\mathrm{B}} T=0.02 \Gamma$, where $\Gamma=\Gamma_{\mathrm{L}}+\Gamma_{\mathrm{R}}$ provides the energy scale, such that the thermal energies are always lower than the level spacing in the dots and much lower than the charging energy.

In the spectral densities $A_{i}(\omega)$ there appear a complex, energy-dependent self-energy term which yields a broadening as well as renormalization of the dot levels. The energy-dependence of the self-energy term is due to the resonant-tunneling processes, and it results in a non-trivial functional form of the spectral functions. On top of this structure Kondo-type resonances may appear as a sharp peaked structure at the position(s) of the electrochemical potential(s). These general effects of resonant tunneling have been found already in the investigations of a single one-level quantum dot $[15,17]$.

We keep the lower level below the electrochemical potentials of the leads such that $\varepsilon_{+} \lesssim-\Gamma$. Tuning the upper level $\varepsilon_{-}=\varepsilon_{+}+\Delta \varepsilon$ allows a crossover from the one-level limit, $\varepsilon_{-} \rightarrow+\infty$, to a fourfold-degenerate level, $\varepsilon_{+}=\varepsilon_{-}$. For the single-dot model the level separation $\Delta \varepsilon$ reflects the actual physical level separation, whereas it is proportional to the coupling strength between the two dots for the double-dot model. In the limit of infinite level separation the spectral density $A_{+}(\omega)$ reproduces results obtained before for a single level [15, 17]. These are the level renormalization and broadening, as well as - for $\varepsilon_{+} \lesssim-\Gamma-$ Kondo-type resonances at the electrochemical potentials of the leads. For a low-lying level this corresponds to the $S=1 / 2$ Kondo problem, however generalized to a nonequilibrium situation if a transport voltage is applied.

When the second level is included, the Kondo peak of the single-level model persists. However, the renormalization effect is stronger and, as long as $\varepsilon_{+}, \varepsilon_{-} \lesssim-\Gamma$, a further peak in the spectral functions appears (dashed curves in Fig. 2). This peak is shifted from the electrochemical potentials by $\Delta \varepsilon$ below (above) the Kondo peak in the spectral function for the lower (upper) level. In the other extreme of zero level separation - fourfold-degenerate level - the resulting spectral functions are equal to each other. At finite temperatures, they display a much more pronounced Kondo peak (solid curve in Fig. 2) than in the single level case - two-fold degenerate level. The appearance of two peaks in the presence of two levels may be interpreted as follows. One peak remains pinned at zero since the spin-degeneracy of the levels continues to lead to a Kondo peak. This result differs from the situation where the spin degeneracy of a single level is lifted by a magnetic field [15]. However, the second peak is of the same origin as the shifted peak in Ref. [15], i.e., it is due to the presence of the other level.

The spectral functions are normalized and having neglected multiple occupancy

$$
\int_{-\infty}^{\infty} d \omega A_{i \sigma}(\omega)=p_{0}+p_{i \sigma} .
$$

Here $p_{0}$ and $p_{i \sigma}$ are the probabilities of an empty dot and that with single electron state $i \sigma$ occupied. This expression allows us to relate differences in the magnitudes of the spectral functions to differences in occupation probabilities, e.g. when the spectral functions corresponding to different levels are compared to each other. 
The bias voltage has the effect of splitting all the resonant peaks in the spectral functions by $e V$, see Fig. 3. The peaked structure of the spectral functions is reflected in a novel structure in the nonlinear conductance, see Fig. 4. Instead of a single zero-bias peak, known from the single level dot, there appear now three sharp resonant peaks between the broad peaks corresponding to the renormalized energy levels. The zero-bias anomaly, characteristic for the single level case remains, but two additional satellite peaks appear at $e V= \pm \Delta \varepsilon$. When the level separation is increased the new peaks decrease in size while the peak at zero bias remains unchanged. When the levels lie above the electrochemical potentials, the nonlinear conductance exhibits a triplet of minima with one minimum at the electrochemical potentials and two satellites on each side.

The systems considered here have not yet been investigated experimentally. Ralph and Buhrman [18] have measured tunneling - enhanced by the Kondo effect - through a localized state of an impurity equivalent to a single one-level dot. Some unexplained features in their results may be due to the existence of the other levels above the electrochemical potentials. The model of a single- or two-level dot with only one electron in it could possibly be realized with the setup used by Tarucha et al. [20]. They argue that they can resolve situations with one or few spin-degenerate levels and one or few electrons in the dot. In particular, they report that a third electron inserted into the dot finds two spin-degenerate levels whose separation can be continuously varied by a magnetic field. In this system, if the gate voltage is fixed near the first or third conductance peak the single- or double-level dot is realized, respectively. The difference should be observable in the nonlinear conductance provided that the zero-bias anomalies can be resolved. The relevant parameters reported in Ref. [20] - the coupling strength (tunneling rate) $\Gamma$, voltage, and temperature - are already close to those used in our calculations.

In conclusion, we have described electronic transport through a two-level quantum dot and a single-level double dot coupled between reservoirs. A real-time diagrammatic technique provides a very general tool for the description of various systems, both in the perturbative and nonperturbative regime. We have found a novel triple-peak structure in the nonlinear conductance of these systems and suggest experiments where it is observable.

$* * *$

We are grateful to L.P. Kouwenhoven, M. Paalanen, and J. Pekola for discussions. This work has been supported by the "Deutsche Forschungsgemeinschaft" as part of "Sonderforschungbereich 195." One of us (GS) also gratefully acknowledges the support through an A. v. Humboldt Research Award of the Academy of Finland and one (TP) the support through a post-graduate Research Fellowship from the Finnish Academy of Science and Letters.

\section{REFERENCES}

[1] P.L. McEuen and L.P. Kouwenhoven, to be published in Nano-Science and Technology, edited by G. Timp, (AIP Press, New York).

[2] M.A. Kastner, Rev. Mod. Phys., 64 (1992) 849.

[3] For a review including more references see H. SCHOELLER, in Mesoscopic Electron Transport, NATO ASI, Series E, eds. L.P. KouWENHOven et al., Kluwer, to be published; and Habilitationsschrift, Karlsruhe (1997).

[4] L.I. Glazman and K.A. Matveev, Pis'ma Zh. Eksp. Teor. Fiz., 48 (1988) 403, [JETP Lett, 48 (1988) 445].

[5] C.W.J. Beenakker, Phys. Rev., B44 (1991) 1646.

[6] Y. Meir, N.S. Wingreen and P.A. Lee, Phys. Rev. Lett., 66 (1991) 3048.

[7] D. Weinmann et al., Europhys. Lett., 26 (1994) 467.

[8] C. Bruder and H. Schofller, Phys. Rev. Lett., 72 (1994) 1076. 
[9] G. Klimeck, G. Chen, and S. Datta, Phys. Rev., B50 (1994) 2316; G. Chen et al., Phys. Rev., B50 (1994) 8035.

[10] C.A. Stafford and S. Das Sarma, Phys. Rev. Lett, 72 (1994) 3590.

[11] P. Pals and A. Mackinnon, J. Phys.: Condens. Matter, 8 (1996) 5401.

[12] J.M. Golden and B.I. Halperin, Phys. Rev., B53 (1996) 1034.

[13] T.K. Ng and P.A. LeE, Phys. Rev. Lett., 61 (1988) 1768.

[14] L.I. Glazman and M.E. Raikh, Pis'ma Zh. Eksp. Teor. Fiz., 47 (1988) 378 [JETP Lett., 47 (1988) 452].

[15] J. König, H. Schoeller and G. Schön, Phys. Rev. Lett., 76 (1996) 1715; J. König et al., Phys. Rev., B54 (1996) 16820.

[16] S. Hershfield, J.H. Davies and J.W. Wilkins, Phys. Rev. Lett., 67 (1991) 3720.

[17] Y. Meir, N.S. Wingreen and P.A. Lee, Phys. Rev. Lett., 70 (1993) 2601.

[18] D.C. Ralph and R.A. Buhrman, Phys. Rev. Lett., 72 (1994) 3401.

[19] D.C. Ralph, C.T. Black and M. Tinkham, Phys. Rev. Lett, 74 (1995) 3241; D.C. Ralph, C.T. Black and M. Tinkham, Phys. Rev. Lett., 78 (1997) 4087;

[20] S. Tarucha, D.G. Austing, T. Honda, R.J. van der Hage and L.P. Kouwenhoven, Phys. Rev. Lett., 77 (1996) 3613.

[21] F.R. Waugh, M.J. Berry, D.J. Mar and R.M. Westervelt, Phys. Rev. Lett., 75 (1995) 705.

[22] T. Schmidt, R.J. Haug, K. v. Klitzing, A. Förster and H. Lüth, Phys. Rev. Lett., 78 (1997) 1544.

[23] H. Schofller and G. Schön, Phys. Rev., B50 (1994) 18436; J. König, H. Schofller and G. ScHÖN, Europhys. Lett., 31 (1995) 31.

[24] The level index $i$ is assumed to be conserved in tunneling as it corresponds to the orbital momentum in vertical quantum dots. For this reason, the quantities $\Gamma_{i j \sigma}^{\alpha}=2 \pi \sum_{k} T_{k i \sigma}^{\alpha *} T_{k j \sigma}^{\alpha} \delta(E-$ $\varepsilon_{\alpha k \sigma}$ ) may be considered to be diagonal in $i, j$.

[25] Y. Meir and N. S. Wingreen, Phys. Rev. Lett., 68 (1992) 2512. 


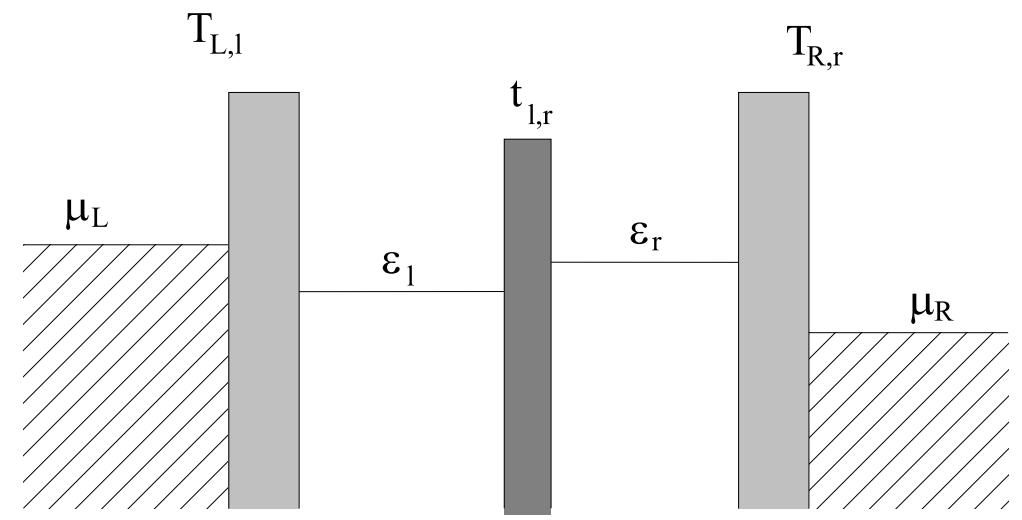

Fig. 1. - Double dot with one level with energy $\varepsilon_{i}$, in each dot. The tunneling matrix elements of the barriers are $T_{\mathrm{L}, 1}$ and $T_{\mathrm{R}, \mathrm{r}}$ for tunneling between the dots and leads and $t_{l, r}$ for tunneling between the dots.

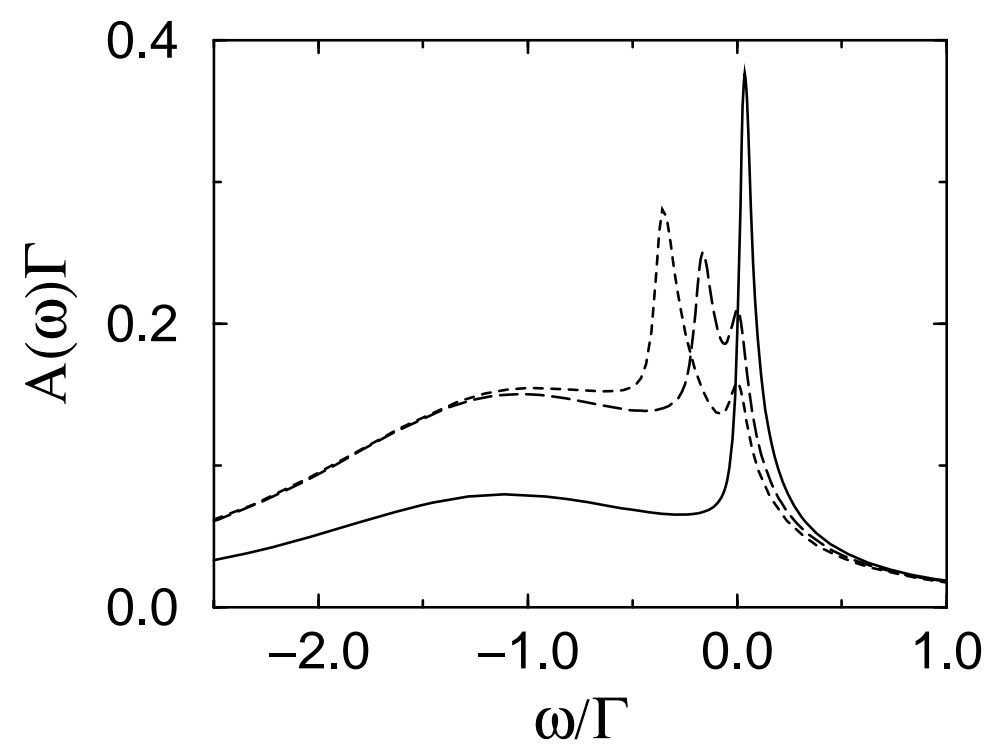

Fig. 2. - Spectral function $A_{+(\sigma)}(\omega)$ with $\varepsilon_{+}=-2.0 \Gamma$ and $\varepsilon_{-}=\varepsilon_{+}+\Delta \varepsilon$. The large Kondo peak of the four-fold degenerate level splits when the degeneracy is lifted. The separation equals the level spacing, $\Delta \varepsilon \in\{0,0.2 \Gamma, 0.4 \Gamma\}$. 


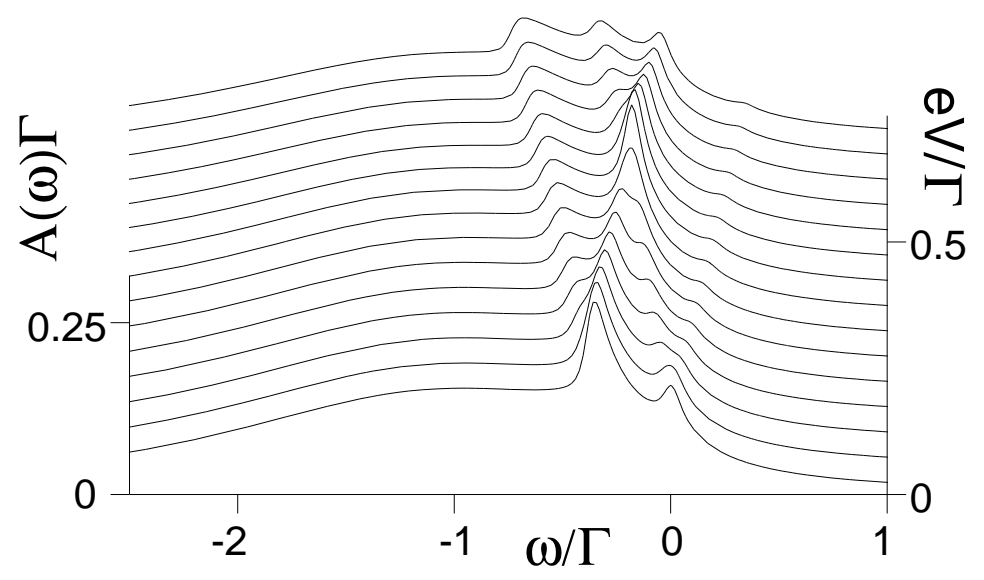

Fig. 3. - Spectral functions $A_{+}(\omega)$ when a bias voltage $V$ is applied. The voltage splits all the resonant peaks by energy $e V$ and the peaks cross each other when $e V=\Delta \varepsilon$.

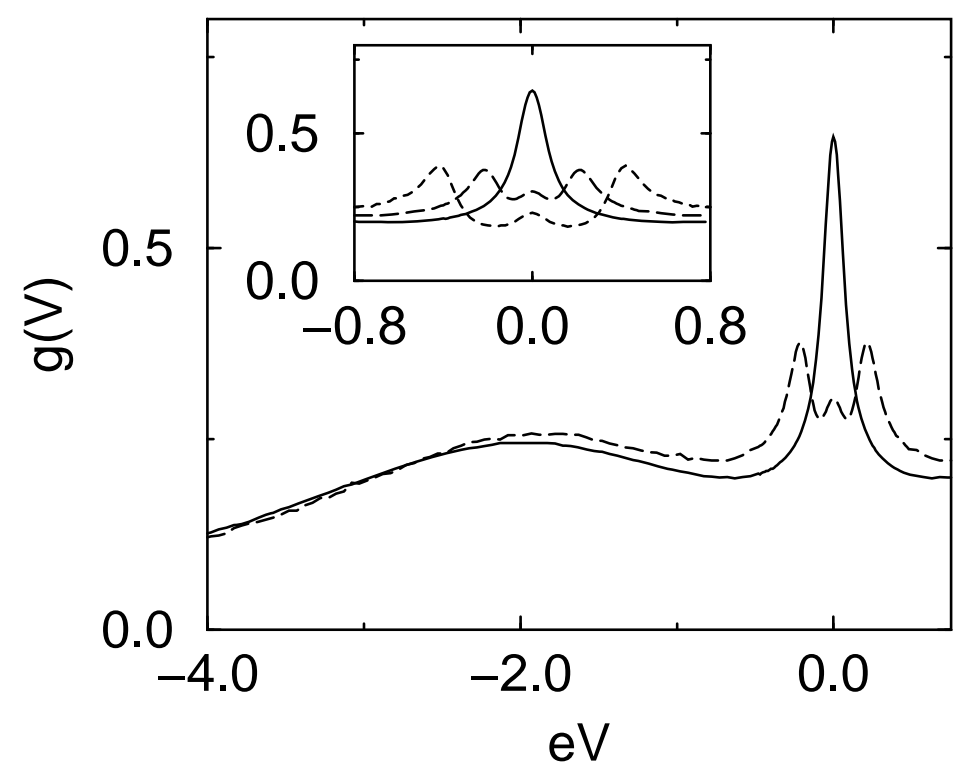

Fig. 4. - The figure shows apart from the broad main peak in the non-linear conductance, which is sometimes referred to as the resonant peak, sharper resonant peaks close to zero. The inset shows the same enlarged. The solid curve is for the four-fold degenerate level. The dashed curves are for split levels, $\Delta \varepsilon=0.2 \Gamma$ (long dashed) and $\Delta \varepsilon=0.4 \Gamma$ (short dashed). The conductance $g(V)$ is expressed in units of $e^{2} / h$. 